\title{
Ubiquitin-Like Modifier-Activating Enzyme ATG7
}

National Cancer Institute

\section{Source}

National Cancer Institute. Ubiquitin-Like Modifier-Activating Enzyme AT G7. NCI

Thesaurus. Code C120969.

Ubiquitin-like modifier-activating enzyme ATG7 (703 aa, 78 kDa) is encoded by the human AT G7 gene. This protein plays a role in both cytoplasm to vacuole transport and autophagy. 